\title{
A Methodical Approach for Detection and 3-D Reconstruction of Brain Tumor in MRI
}

\author{
Sayali Lopes \\ M.E. Student, Dept. of Computer Engg., \\ St. Francis Inst. of Technology, \\ Mumbai, India
}

\author{
Deepak Jayaswal, PhD \\ Prof., Dept. of Electronics and \\ Telecommunication Engg., \\ St. Francis Inst. of Technology, \\ Mumbai, India
}

\begin{abstract}
It always takes a skilled neurologist to detect a tumor in the MRI scans, which the numerologist does with the naked eye. Doctors have had only 2D cross sectional images for viewing the tumor in the MRI scans. This research presents a method for automatic tumor detection with an added feature of reconstructing its 3D image. The research involves implementation of various steps of detecting and extracting the tumor from the 2D slices of MRI brain images by Seeded region growing technique along with automatic seed selection and designing software for reconstructing 3D image from a set of $2 \mathrm{D}$ tumor images. The seeded region growing method is very attractive method for semantic image segmentation which involves high level knowledge of image components during the seed selection procedure. The volume of the tumor is also estimated based on the computation of these images to assist the radiologist.
\end{abstract}

\section{General Terms:}

Magnetic Resonance Imaging

\section{Keywords}

Brain Tumor, Segmentation and 3D visualization.

\section{INTRODUCTION}

Brain tumors are a group of abnormal cells that grow in or around the brain. They can destroy the brain cells or cause indirect damage that can cause swelling in the brain and increase the pressure within the skull. Tumors can appear malignant or benign. Malignant tumors grow rapidly and often invade or crowd healthy areas of the brain. Benign tumors do not contain cancer cells and usually grow slow. A brain tumor can be classified based on i) where the tumor is located, ii)the type of tissues involved and iii) whether it is benign or malignant. The primary cause of brain tumors is unknown. However environmental and genetic factors may play a role or priori exposure to therapeutic irradiation as a child may be a contributing cause in very few patients. Brain tumor symptoms include headaches, nausea, seizures, behavior changes, memory loss, vision and hearing problems. Many treatment options are available on different criteria. Treatment options depend on size and type of tumor, growth rate and general health of the patient. These treatment options generally include radiation therapy, chemotherapy and surgery.

Magnetic Resonance Imaging (MRI) is an imaging modality used in radiology for diagnostic purpose. It is technique that uses nuclear magnetic resonance to form cross sectional images of the human body for diagnostic purposes. Information about the complex anatomic structures of human body is obtained from MRI. These complex 3D anatomic structures are presented as $2 \mathrm{D}$ images. Thus $3 \mathrm{D}$ volumetric data is displayed in the form of $2 \mathrm{D}$ projected slices may result in loss of important information that leads to erroneous interpretation of results [1]. This has lead to the research in the fields of 3D reconstruction of volumetric data from 2D slices. 3D representation of volumetric data is closer to reality and helps the radiologist to interpret the results correctly, thus helping in correct diagnosis of the disease.

The 2D images cannot exactly convey the complexities of human anatomy and hence interpretation of such complex anatomy in $2 \mathrm{D}$ images requires a trained person. Radiologists are trained to interpret the complex anatomy in $2 \mathrm{D}$ images. The radiologist sometimes finds it difficult to communicate the interpretations to physician who may have difficulty in imaging the 3D anatomy. This leads to the need for reconstructing the $2 \mathrm{D}$ cross sectional image data of the tumor into 3D data. The 3D visualization of the tumor data helps in better understanding of the topology and the shape of the tumor. The tumor information which is extracted is helpful in staging of the tumor and helps the physician in surgical planning. Therefore, how to reconstruct a trustworthy surface from the sequential parallel $2 \mathrm{D}$ cross sections becomes a crucial issue in biomedical 3D visualization [2].

The main task of reconstruction is divided into subtask. In the first step preprocessing is performed on the images to improve the quality of the images. Then segmentation algorithm is performed to segment the tumor region. After segmenting the tumor region in order to provide smooth surface smoothening operation are performed on the slices. In the next step graphing functions are used for volume visualization to reconstruct the data into a 3D data. Last the volume of the tumor is calculated.

The remnant part of this paper is arranged as follows. Section II gives the detailed information of segmentation techniques that are available for use. In section III, overview of entire proposed system is discussed followed by the implementation details and conclusion in Section IV, and Section V respectively.

\section{REVIEW OF LITERATURE}

The use of MRI as a modality for viewing brain tumor has lead to research for improving the detection and evaluation of the abnormalities. Despite the difficulties that obstruct the development of brain tumor segmentation algorithms, there are few successful attempts that give accurate results. The data used by these algorithms is obtained from different sources. The data sources include opposite contrast effects on the image tissues intensities on T1-weighted and T2-weighted images, knowledge of different matter distribution and anatomic structures. Clark et al. [4] proposed one such method where the algorithm removes extra-cranial tissues using fuzzy c-means clustering. Statistical analysis is performed to locate the abnormal regions inside the intra-cranial tissue. The algorithm 
is passed through training phase and the results obtained have a relatively large error. In [1] Karunakar et al. presents a method for 3D reconstruction. The paper involves implementing various steps of extracting the tumor from the $2 \mathrm{D}$ slices of MRI brain images by OTSUs threshold technique and various morphological operations and designing software for reconstructing 3D data from a set of $2 \mathrm{D}$ images. In this paper the author does the task providing the seed point for thresholding manually. This is a time consuming task. Thus the execution time of the algorithm increases. In [2] Megha et al. proposed an effective and efficient approach to 3D reconstruction of brain tumor and estimation of its volume from a set of two dimensional (2D) cross sectional magnetic resonance (MR) images of the brain. The proposed 3D reconstruction approach can generate an accurate $3 \mathrm{D}$ model in less amount of time and thus can assist the radiologist in the diagnosis, identifying the stage of the tumor and treatment planning. In [5] $\mathrm{N}$ Moon et al. proposes automatic brain segmentation method that uses an Expectation Maximization algorithm. The algorithm estimates the probability distribution of the tissue classes which consist of gray matter, white matter cerebrospinal fluid, and tumor. Prior information and assumption are required to do the segmentation. In [3] S. Koompairojn makes use of multiple source data to implement an automatic algorithm. The algorithm is based on Evidence theory to combine knowledge obtained from different sources. In [15] S. Ghanavati presents an automatic algorithm for detection of tumor by fusing the information extracted from different MRI modalities which include T1, T2, and T1 with gadolinium contrast. In Corso et al. [17] a Bayesian formulation is integrated into segmentation by a weighted aggregation algorithm to identify GBM (glioblastomamultiforme) brain tumors. In this voxel intensities in a neighborhood are used to compute an affinity among the surrounding voxels to train the segmentation model parameters and then classify them. In [16], N. Gordillo et al. developed a set of fuzzy rules to segment the tumor from the MRI images with expert intervention and analysis of pixels using histogram. Ratan et al. [18] uses watershed algorithm for image segmentation to get an approximate shape of the tumor. In [19], T. Wang et al. used a Bayesian classifier to identify the location of the tumor and a fluid vector flow algorithm is used to estimate the shape of the tumor. In [16] Nelly Gordillo presented a review of the most relevant tumor segmentation methods. According to [21] automatic and semi-automatic segmentation methods have gained the importance due to their accuracy in identification but the end systems are used by the physicians therefore find a surprising lack of compatibility between computer vision based systems and low level segmentation methods. These approaches which lack standardized procedures still cannot gain acceptance from the pathologist for clinical task. These approaches need to be compared with real world medical issues to address problems of segmentation with best suitable approaches. In [21] comparison between watershed, histogram thresholding, global thresholding, fuzzy c-mean and seeded region growing based brain tumor segmentation methods is done. Statistical and visual analysis performed on the methods show that seeded region growing method is found to be the best segmentation method. The major issue in the seeded region growing method is to identify an appropriate homogeneity criterion to select a seed point. The paper introduces a homogeneity criterion for selecting a proper seed point.

\section{PROPOSED WORK}

This paper proposes a method for detection and 3Dreconstruction of brain tumor. The following steps describe the overall architecture of the processing of the system. First it starts with the loading of the MRI brain image, which is considered as an input image. The preprocessing of this input image takes place i.e. the enhancement of the image. In the segmentation stage, first the tumor pixels are identified then extracted. The tumor volume is calculated and the $3 \mathrm{D}$ volume data is displayed. The flow of the proposed systems is given in fig. 1. The steps involved are explained below.

\subsection{Enhancing the Image: Data Preprocessing}

The images obtained from a MRI scan are divided into T1 weighted and T2 weighted images. Depending on the pulse sequence used and the type of tissue in the image region of interest, signal in MR images can be high or low (bright or dark). The tissues in $\mathrm{t} 1$ and $\mathrm{T} 2$ weighted images appear bright or dark depending on the scan. In T1 weighted images the gray matter and tumor is shown in black while the white matter is shown in gray. In T2 weighted images the gray matter and tumor are shown in gray while the white matter is black. Using the above information tumor can be identified on the basis of the intensity of the tissues. For our research work T2 weighted images are used. As stated above tumor in T2 weighted images are seen in gray color, with the help of a radiologist the manual segmentation of tumor was done. It was found out that the gray level intensities of the tumor lie in the range 130 to 190 . The scans include information about the patients and the skull boundary whose pixel intensities also fall in the range of 100 to 190, which can affect the automatic tumor detection process. Due to these reasons preprocessing needs to be done on these scans.

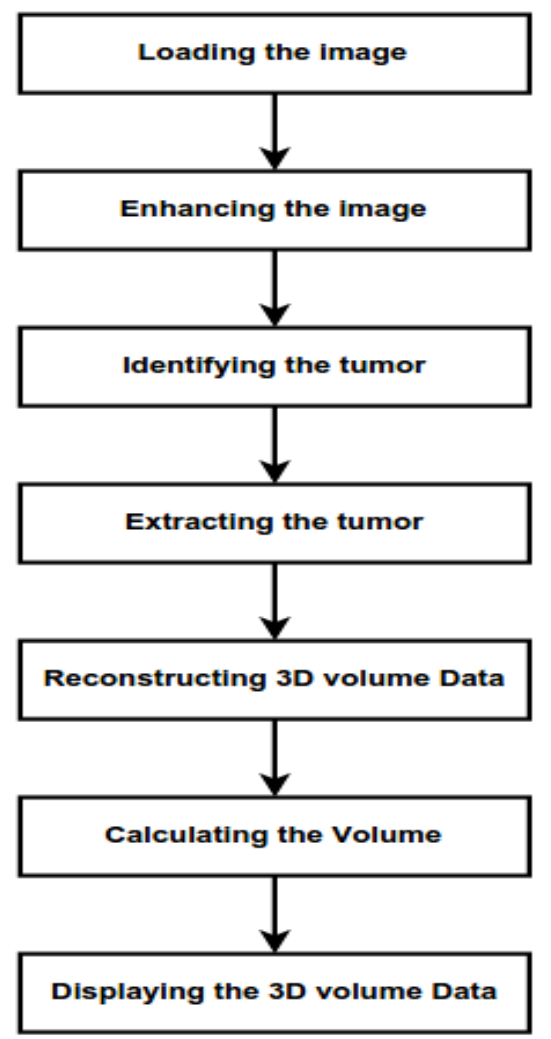

Fig 1: Flowchart of the proposed approach 

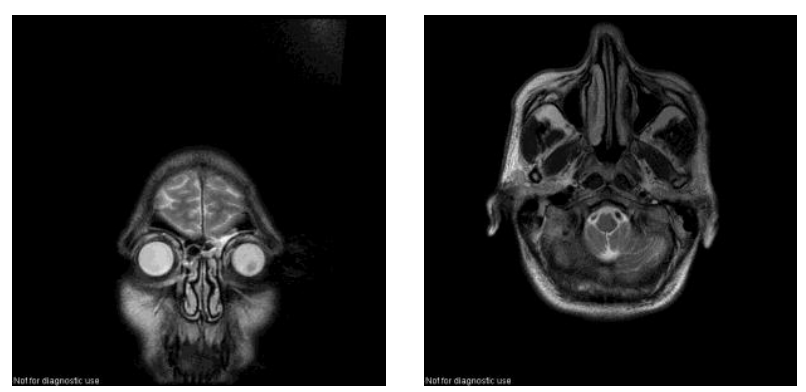

Fig 2:a)Added text in the left corner and b) skull boundary along the edge

Moreover, compared with Fig 2b, in Fig 2a the skull is not in the central. This biased position would bring biased computation when detecting tumor location, as the scans will be loaded in a set and the tumor location will be marked on different position for different slices for a particular patient and will be problematic while reconstructing the $3 \mathrm{D}$ tumor. So before detecting the tumor, we have to

1. Remove the text by pruning the image and

2. Remove the surrounding black portion and keep the skull portion.

We consider 24 slices of $512 \times 512$ pixels in trans-axial view. We are interested in only the brain region so the surrounding black portion of the image is removed. As we know that the intensity of skull portion and the written artifacts lies between 100-190. Using this criterion the black portion removing algorithm is used to remove the black portion. It is seen that artifacts about the patient are in the left hand side corner of the image which also lies in the range 130-190 which can affect the black portion removal algorithm and the artifacts won't get removed. To avoid this we prune the image by removing the lower 12 pixels using the pruning algorithm followed by the black portion removing algorithm. The algorithm is as follows.

\section{Pruning algorithm}

Step1: Load the image $\mathbf{J}$

Step 2: Define a matrix (where rows of image $J=1: 500$ for all columns)

Step 3: Store the matrix in J2

Step 4: Display the image J2

\section{Algorithm to remove black portion}

Step 1: Acquire the image J2

Step 2: Find the location (indices) of the elements in J2 that are greater than or equal to $100 \mathrm{x}$

Step 3: Represent the rows and columns of the element by $[a, b]$

Step 4: Find out the smallest element in $[\mathrm{a}, \mathrm{b}]: \mathrm{m} 1=\min (\mathrm{a})$ $\mathrm{n} 1=\min (\mathrm{b})$

Step 5: Find out largest element in $[\mathrm{a}, \mathrm{b}]: \mathrm{m} 2=\max (\mathrm{a})$ $\mathrm{n} 2=\max (\mathrm{b})$

Step 6: Create a image J3 (m1:m2, n1:n2)

Step 7: Display the image J3

The pre-processed image is shown in Fig. 3.

\subsection{Identifying the tumor:}

The tumor pixel intensities are ranging from 130 to 190 on a scale of 0 to 255 . So threshold range was given as 130 to 190 .

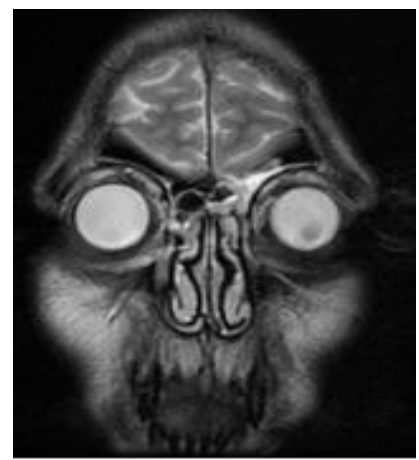

Fig 3: Reshaped image

\subsubsection{Define the tumor shape: Selection of a seed point}

When detecting tumor manually, one could check the grey degree roughly. And in the automatic procedure, we define the tumor with grey degree between 130 and 190. But this single rule will find many areas having preferred grey but actually they are "false tumor". The nerves tissues as seen in Fig 3 has grey degree in the interval of 130 and 190, however, it shouldn't be a tumor because it is so small and it could be other normal tissue in the brain. The minimum size of the tumor is observed to be 1.25 inches in diameter which is approximately equal to 120 pixels [23]. To avoid false detection of the tumor we use a filtering rule: area of the square. Suppose we detect a pixel having gray degree between 130 and 190, we then check the square with this pixel the centroid (seen in Fig. 4). The radius of the square is defined by a proper value. A large radius will omit the tumor and cause 'True Negative' detection, and a small radius will give results as false tumor and cause 'False Positive detection'. In our approach, we use 5 as the radius as the smallest of the tumor can occupy $10 x 10$ pixels.

We check each pixel within the square that, if each pixel having grey degree between 130 and 190 then it will be marked as tumor, else moves on to next pixels.

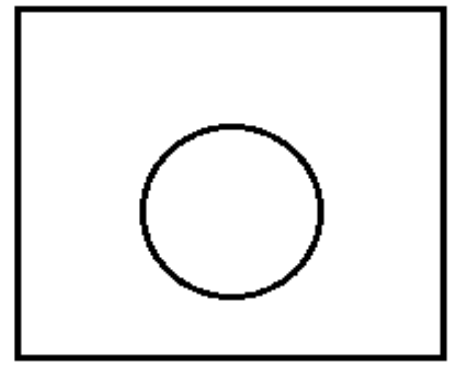

Fig. 4: Square of a Pixel: the circle is the pixel having grey degree in 130 and 190, and the square surrounding it is the area that needs to check 


\subsubsection{Scan the pruned image}

We apply this rule to scan the whole image pruned in Step 1. Since we believe that the tumor will occupy an area with $10 \times$ 10 pixel (Because the radius is 5, then the length of the square side is 10), we could start from the 5th pixel in both vertical axis and vertical axis, and stop at the last 5th pixel. If detecting any square satisfying the filtering rule in section 3.2.1, we stop scanning the current image and invoke the next image, repeating the above Steps.

\subsection{Extracting the tumor: Seeded Region Growing:}

After loading the image sequence and locating the seed pixels, region growing algorithm is applied. The region is then grown from the seed point to adjacent seed points using the region membership criterion which is the pixel intensity for our approach. We use the 8 connected neighbourhoods for our pixels adjacent relationship. The output is a binary image. Sometimes, some of the pixels in the tumor region do not satisfy the threshold criteria leaving holes in that region, thus not extracting the tumor region properly. If such holes are found flood fill algorithm which is a region filling algorithm is use to overcome the problem [20].

\subsection{Reconstructing the 3D volume data:}

After the tumor region is extracted smoothening operations are performed to smooth the data. Before moving to the reconstruction of $3 \mathrm{D}$ tumor the binary images which are obtained in the previous step need to be smoothened. Smoothening is performed to aid the process of reconstruction.

The reconstruction technique is categorized into following steps.

1. Graphing volume data usually requires knowledge of the range of both the coordinates and the data values.

2. Select an appropriate plotting routine. Here the function connects to the points that have the specified value, the way the contour lines are connected to the point of equal elevation. Arrays $\mathrm{x}, \mathrm{y}$ and $\mathrm{z}$ are created to define the coordinates for the volume data.

3. The final answer which is returned by the function is passed to the patch command. The patch function acquires the final value. The patch function creates the patch graphic object.

4. The face and edge color is set.

5. Specifying the view: in this step we define the view. By using viewing techniques the information conveyed by a complex 3D graph can be greatly enhanced.
6. Adding lights: In this step we add lighting and specify coloring. The effective means to enhance the visibility of the surface shape is best achieved by lighting. Lighting provides a three dimensional perspective to volume graphs.

\subsection{Displaying the 3D volume:}

Here in this phase the tumor is displayed in a 3D view. The creation of graphical representation of data sets that are defined on three dimensional grids can be termed as volume visualization. The MRI data is a scalar data. The data contains a number of slice planes taken through a human body. The scalar volume data typically is composed of a 3D array of data and 3 co-ordinate arrays of the same direction which specify the $\mathrm{x}, \mathrm{y}$ and $\mathrm{z}$ coordinate of each data point.

\subsection{Calculating the volume:}

The volume of the tumor is calculated by calculating the area once each slice is binarised and then multiplying it by scale/distance of the images in the $\mathrm{x}, \mathrm{y}$ and $\mathrm{z}$ direction. The following formula is used.

Tumor volume $=$ total area $* \mathrm{x} * \mathrm{y} * \mathrm{z}$

\section{PERFORMANCE STUDY}

This section introduces performance metrics and discusses the result obtained.

The experimental data set of 240 images has been obtained from the Tata Memorial hospital for Cancer Research, Mumbai. The Measure and Metrics used to evaluate the performance of the proposed method are derived from the comparison of the segmented region with its corresponding ground truth. Radiologist's manual delineation of tumor in each slice is treated as the ground truth.

\subsection{Performance results}

The test database set consists of 240 MRI images of 10 patients. In Table 1 the overall result of tumor detection are shown. The columns show the ground truth and the rows show the test results. The presence oftumor is correctly detected in $90 \%$ cases and the absence of it was correctly classified in $100 \%$ cases.

Table 1

\begin{tabular}{|l|c|c|}
\hline & Tumor present & Tumor absent \\
\hline Test positive & $90 \%$ & $100 \%$ \\
\hline Test negative & $10 \%$ & $0 \%$ \\
\hline
\end{tabular}




\subsection{Results:}

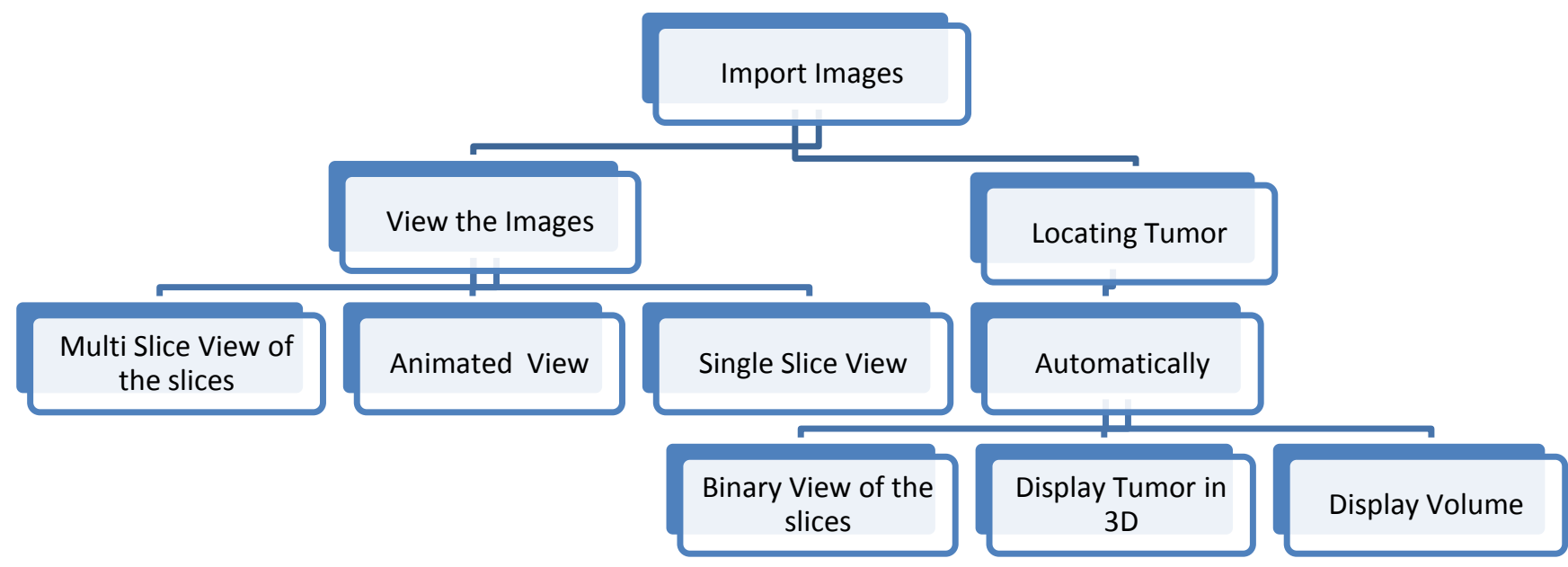

Fig 5: Flow sheet explaining the control flow of the software

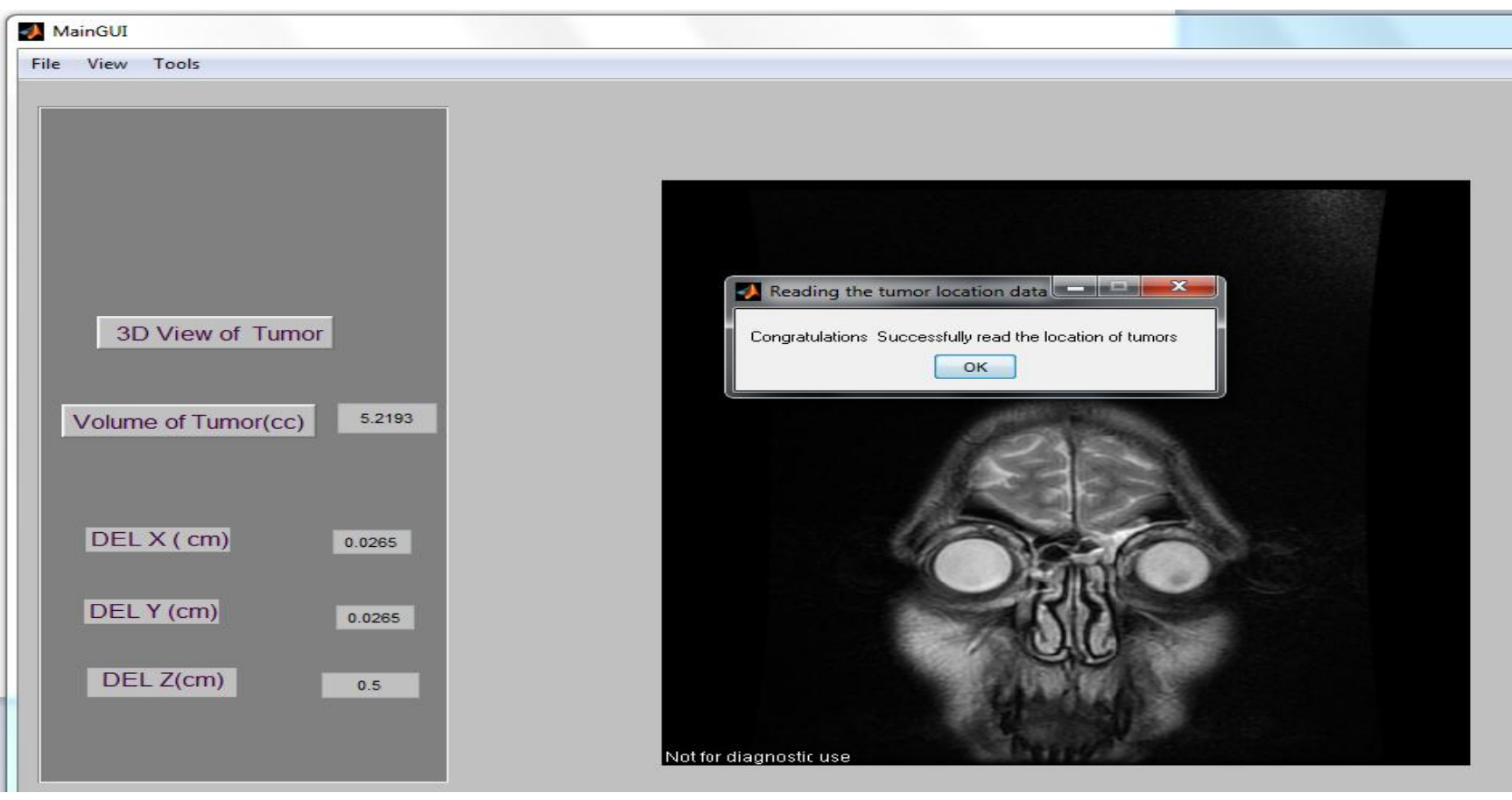

Fig 6: Tumor Located Automatically 


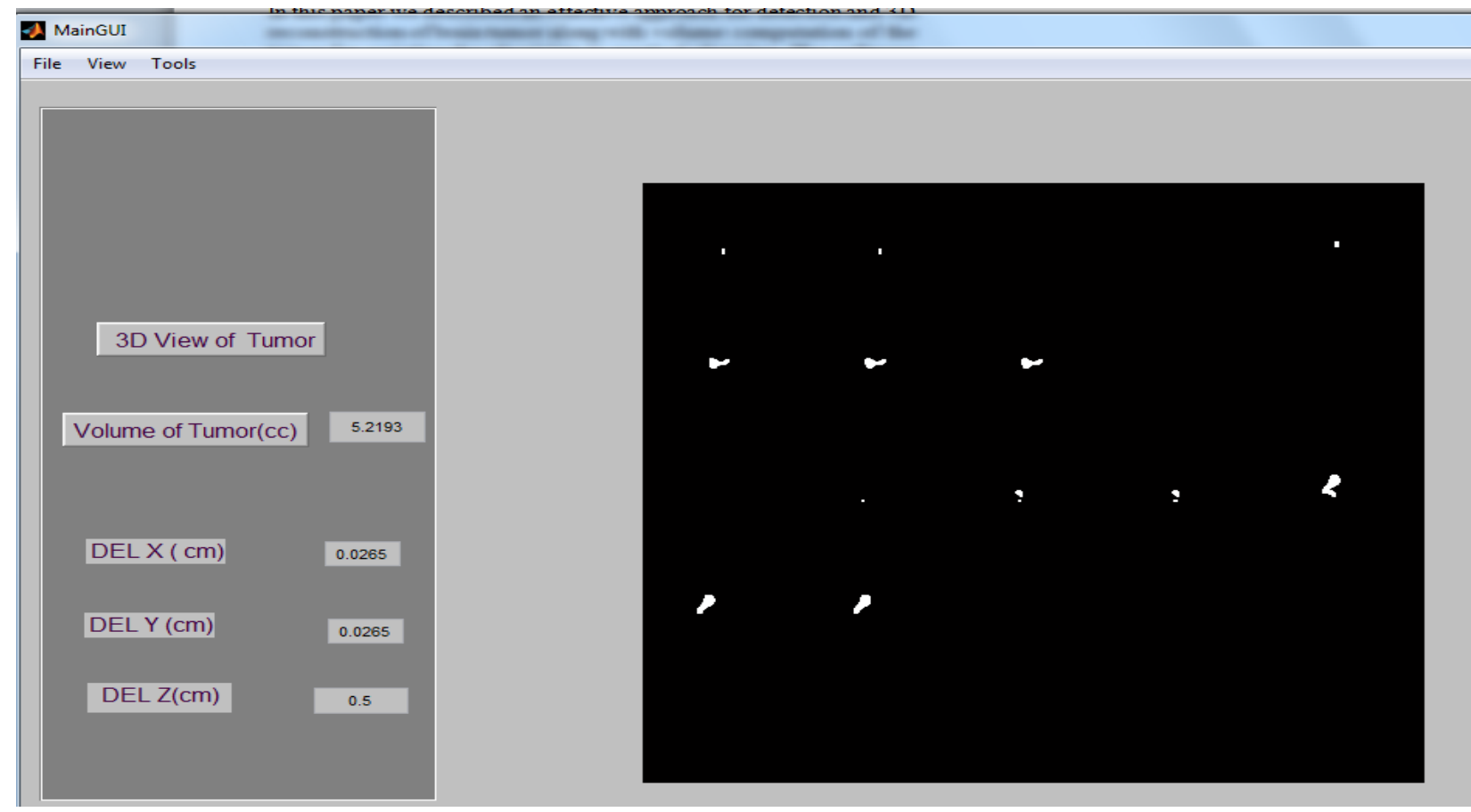

Fig 7: Binary view of the Tumor

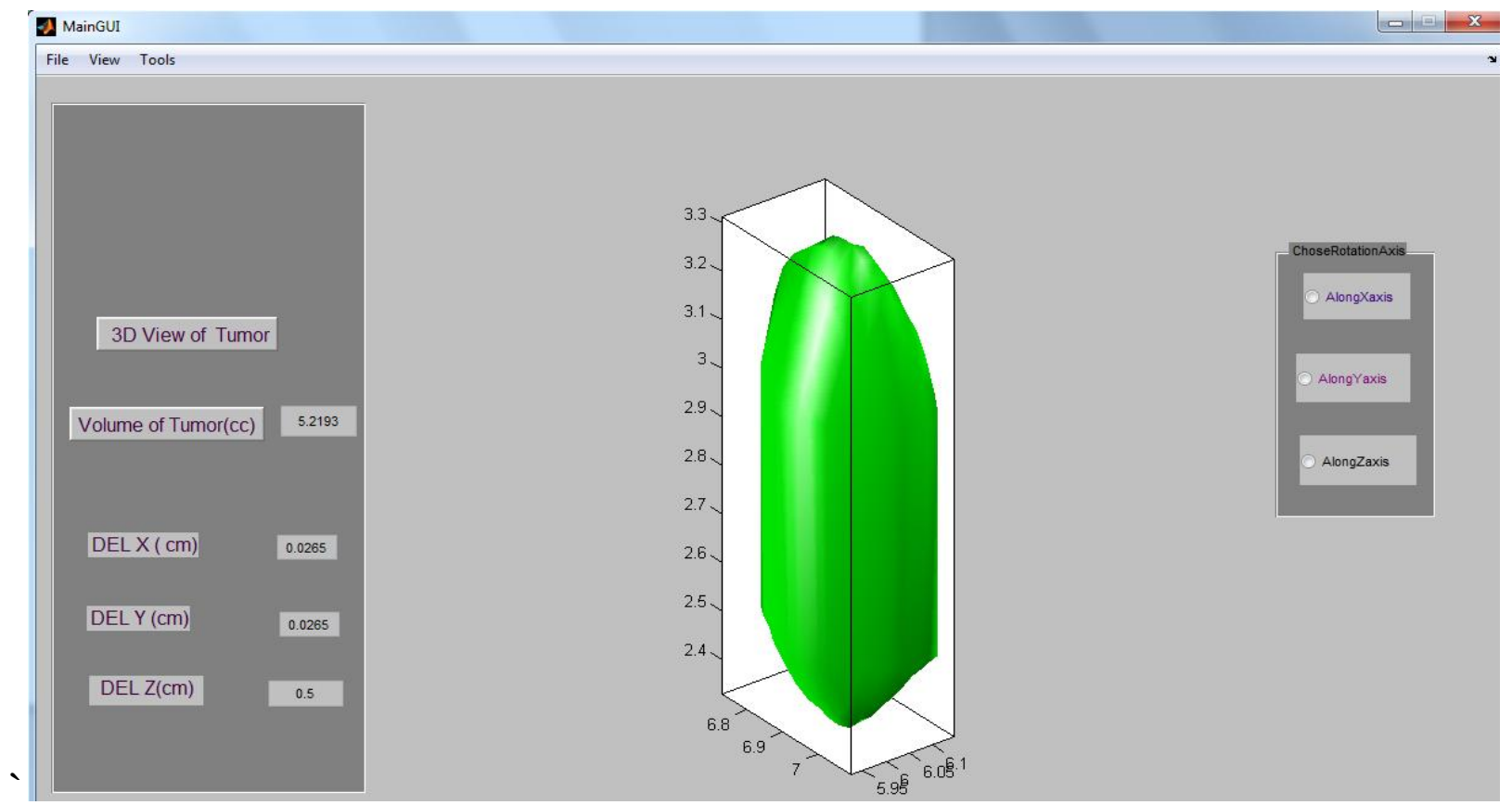

Fig 8: 3D View of Tumor, Volume of Tumor, Volume $=5.219$ cubic $\mathbf{c m}$.

\section{CONCLUSION}

Detection brain tumor in MRI in a fast, accurate, and reproducible way is a challenging problem. Automatic image segmentation has become very important aspect for realizing content based image description. Seeded region growing algorithm is robust, rapid and effective as compared other segmentation algorithm. In this paper we described an effective approach for detection and 3D reconstruction of brain tumor using the seeded region growing algorithm using automatic seed selection along with volume computation of the tumor for assisting the physician in surgical planning. It is very difficult to classify a tumor as benign or malignant. There are a number of pathological features which contribute in determination of the tumor whether it is benign or malignant. This will be the topic for future research. Further, in future we would work on implementing the software to detect tumors in other parts of the body like lungs, throat and pelvic. 


\section{REFERENCES}

[1] Anantatamukala, A. Gole, A. and Karunakar, Y. A Systematic Algorithm for 3-D Reconstruction of MRI based Brain Tumors using Morphological operators and Bicubic Interpolation. Proceedings of $2^{\text {nd }}$ International Conference on Computer Technology and development(ICCTD). (2010), 305-309.

[2] Arakeri, M. and Reddy, G. An Effective and Efficient Approach to 3D Reconstruction and quantification of brain Tumor on Magnetic Resonance Images. IEEE Trans. Signal Processing, Image Processing and Pattern Recognition. (June 2013), 111-128.

[3] Koompairojn, S. Petkova, A. Hua, K. and Metarugcheep, P. Semi-automatic Segmentation and Volume Determination of Brain Mass-like Lesion. 21 ${ }^{\text {st }}$ IEEE International Symposium on Computer Based Medical Systems.(2008), $35-40$.

[4] Clark, M. Lawrence, L. Golgof, D. Velthuizen, R. Murtagh, F. and Silbiger, M. Automatic Tumor Segmentation Using Knowledge based technique. IEEE Trans. Medical Imaging. (April 1998), 158-167.

[5] Moon, N. Bullitt, E. Leemput, K. and Gerig, G. Modelbased brain tumor segmentation. Proceedings of IEEE Int Conf. Pattern Recognition. (2002), 526-531.

[6] Kharrat, A. Messaoud, M. Benamrane, N. Abid, M. Detection of Brain Tumor in Medical Images.Proceedings of IEEE Int. Conf.Signals, Citcuits and Systems. (2009), 1-6.

[7] Islam, R. Mamun, A. bhuiyan, M. Rahman, S. Segmentation and 3D visualization of volumetric Image for Detection of Tumor in Cancerous Brain. Proceedings of IEEE Int. Conf. on Electrical and Computer Engineering. (December 2012), 863-866.

[8] Natrajan, P. Krishnan, N. Kenkre, N. Nancy, S. Singh,B. Tumor Detection using threshold operation in MRI Brain Images. Proceedings of IEEE Int. Conf. on Computational Intelligence and Computing Research. (2012), 1-4.

[9] Kumar, P. Kumar, N. and Sumithra, M. Tumor Detection in Brain MRI Using Improved Segmentation Algorithm. Proceedings of IEEE Int. Conf. on Computing, Communications and Networking Technologies. (July 2013), 1-7.

[10] Xu, T. Mandal, M. Automatic Brain Tumor Extraction from T1- Weighted Coronal MRI Using Fast Bounding Box and Dynamic Snake. Proceedings of IEEE Int. Conf. on EMBS. (August- September 2012), 444-447.

[11] Hamamci, A. Kucuk, N. Karaman,K.Engin, K.Unal,G. Tumor-cut: Segmentation of Brain Tumors on Contrast
Enhanced MR Images for Radiosurgery Applications. IEEE Trans. Medical Imaging. (March 2012), 790-804.

[12] Karimaghaloo, Z. Shah, M. Francis, S. Arnold, D. Collins, $\mathrm{D}$ and Arbel, T. Automatic Detection of GadoliniumEnhancing Multiple Sclerosis Lesions in Brain MRI Using Conditional Random Fields. IEEE Trans. Medical Imaging. (June 2012), 1181 - 1194.

[13] Verma, N. Muralidhar, G. Model-driven, probabilistic level set based segmentation of magnetic resonance images of the brain. Proceedings of IEEE Int. Conf. on EMBS. (AugustSeptember2011), 2821-2824.

[14] Kazerooni, A. Ahmadian, A. Segmentation of Brain Tumors in MRI Images Using Multi-Scale Gradient Vector Flow. Proceedings of IEEE Int. Conf. on EMBS. (AugustSeptember 2011), 7973-27.

[15] Ghanavati, S. Junning, L. Ting, L. Babyn, P. Doda, W. Lampropoulos, G. Automatic brain tumor detection in Magnetic Resonance Image. 21st IEEE International Symposium on Computer Based Medical Systems. (May 2012), 574-577.

[16] Gordillo, N. Montseny, E and Sobrevilla, P. New fuzzy approach to brain tumor segmentation.Proceedings of IEEE Int. Conf. on Fuzzy Systems. (2010), 1-8.

[17] Corso, J. Sharon, E. Dube, S. El-Saden, S. Sinha, U. and Yuille, A. Efficient multilevel brain tumor segmentation with integrated bayesian model classification. IEEE Trans. Med. Imaging. (2008), 629-640.

[18] Ratana, R. Sharma, S. Sharma, S. Brain tumor detection based on multi-parameter MRI image analysis. ICGST Int. J. Graphics, Vision and Image Processing.(2009), 9-17.

[19] Wang, T. Cheng, I and Basu, A. Fully automatic brain tumor Segmentation using a normalized Gaussian Bayesian classifier and3D Fluid vector flow. Proceedings of IEEE Int. Conf. on Image Processing. (2010), 2553-2556.

[20] Soille, P. Morphological Image Analysis: Principles and Applications, Springer-Verlag, 1999, pp. 173-174

[21] Fan, J. Zeng, G. Body, M. Hacid, M. Seeded region growing: an extensive and comparative study. Pattern Recognition Letters. Elsevier. (2005), 1139-1156.

[22] Measurement of Tumor "Size" in Recurrent Malignant Glioma: $\quad 1 \mathrm{D}, \quad 2 \mathrm{D}, \quad$ or $3 \mathrm{D}$ ? http://www.ajnr.org/content/26/4/770/T1.expansion.html $25 / 03 / 2015$

[23] Brain Cancer In-Depth Report: http://www.nytimes.com/health/guides/disease/brain-tumoradults/print.html 25/03/15 